\title{
Multiple Myeloma in Portugal: Burden of Disease and Cost of Illness
}

\author{
Manuel Neves ${ }^{1} \cdot$ Fernanda Trigo $^{2}$ - Bergantim Rui ${ }^{2}$. Cristina João ${ }^{1} \cdot$ Paulo Lúcio $^{1} \cdot$ Neves Mariana ${ }^{3}$ João Mendes ${ }^{4}$. \\ Hugo Pedrosa ${ }^{3,6}$ (1) $\cdot$ Catarina Geraldes ${ }^{5}$
}

Accepted: 24 December 2020 / Published online: 31 January 2021

(c) The Author(s) 2021

\begin{abstract}
Background Multiple myeloma (MM) is the second most common hematological cancer worldwide and has significant morbidity and mortality and is increasing in incidence. While MM management costs are considerable, specific economic data at the country level remain scarce.

Objective This study assesses the burden and cost of MM in Portugal from the perspective of the National Health Service (NHS) to support the definition of health policies, resource allocation and patient care.

Methods Developed by the Portuguese Multiple Myeloma Group, this study considers the most recent available data. Burden of disease was measured using disability-adjusted life-years (DALYs). The cost of MM was estimated using a prevalencebased model that estimated direct costs for the NHS considering all costs associated with diagnosis, hospitalizations, surgeries, emergency visits, medical appointments, drugs and transportation. Costs were quantified based on the diagnosis-related group funding price, except for drug usage, which was calculated using the average hospital product stock price.

Results The burden of disease attributable to MM for 2018 was estimated at 8931 DALYs: 8570 resulting from premature deaths and 361 from disability. Average yearly direct costs per patients with MM amounted to $€ 31,449$ (year 2018 values). Total direct costs are estimated at $€ 61$ million per year.

Conclusions The mortality rate in MM means that most DALYs are due to years of life lost rather than years lost due to disability. This study generates comprehensive data on the burden and cost of MM in Portugal and provides updated insights into the costs associated with the management of MM.
\end{abstract}

\section{Introduction}

The financial burden associated with cancer is increasing worldwide. The emergence of new drugs (e.g., monoclonal antibodies, checkpoint inhibitors, chimeric antigen receptor T-cell therapy) has exponentially increased the

Hugo Pedrosa

hugo.pedrosa@iqvia.com

1 Hemato-Oncology Unit, Hematology Department, Clinical Center of the Champalimaud Center for the Unknown, Lisbon, Portugal

2 Hematology Department, Centro Hospitalar S. João, Porto, Portugal

3 IQVIA Solutions Portugal, Lisbon, Portugal

4 Janssen Portugal, Lisbon, Portugal

5 Hematology Department, Centro Hospitalar e Universitário de Coimbra, Coimbra, Portugal

6 Lagoas Park, Edifício 3, Piso 3 Porto Salvo, 2740-266 Oeiras, Portugal financial impact of cancer, with a global estimated cost of \$US150 billion in 2020. There is also an impact in terms of loss of productivity, as reported in BRICS (Brazil, Russia, India, China, South Africa) countries, which lost \$US46.3 billion in 2012 due to cancer deaths [1,2].

In 2017, the annual cost of cancer treatment in Portugal was $€ 867$ million, representing around $5 \%$ of the total health expenditure, with an average cost of $€ 84$ per capita. Antineoplastic drugs were the main component of this cost, making up $31.5 \%$ of the total. Knowing the cost of cancer treatment is crucial for the management of the health system and its associated units. The impact of cancer on mortality and morbidity as well as the loss of productivity and the rising costs of treatment make cancer an economic priority [3].

Multiple myeloma (MM) is the second most common hematological cancer worldwide, with a median age at diagnosis of 65-70 years [4]. With the development of new therapeutic options, life expectancy has increased significantly, and MM has gone from being an untreatable 


\section{Key Points for Decision Makers}

Multiple myeloma has a significant burden in Portugal, being responsible for 8931 disability-adjusted life-years (DALYs) and $€ 61$ million in direct costs in 2018.

A major share of the cost is associated with drugs, with $77 \%$ of these attributable to lenalidomide and bortezomib. These costs are expected to increase because of the use of novel drugs with better efficacy and increased overall survival.

This study generates comprehensive data on the burden and cost of multiple myeloma in Portugal and provides updated insights into the costs associated with its management.

ailment to one that, while still not curable, is treatable through mostly outpatient treatment regimens [5].

MM is characterized by the clonal proliferation of plasma cells that produce a monoclonal immunoglobulin [6]. The proliferation of these clonal plasma cells in the bone marrow causes skeletal damage, a hallmark of MM, with up to $95 \%$ of patients developing osteolytic bone lesions at some point during the course of the disease [7]. MM is also characterized by anemia and renal and immunological dysfunction. These complications may severely reduce the quality of life and the life expectancy of patients with MM [8].

International guidelines for the pharmacological treatment of MM include bortezomib-based and thalidomideor lenalidomide-based protocols as front-line therapies for patients with MM. For patients who meet the defined inclusion criteria (fit patients aged $<70$ years), the guidelines recommend autologous stem-cell transplant (ASCT), preceded by high-dose melphalan conditioning and followed by lenalidomide maintenance as the standard treatment [4].

The 2016 Global Burden of Disease Study reported 138,509 incident cases of MM worldwide, 98,437 deaths, and 2.1 million disability-adjusted life-years (DALYs). This is a measure that indicates the overall burden of a disease and allows comparison with other diseases with different characteristics and impacts in terms of mortality or chronic disability. The incidence of MM has reportedly increased by $126 \%$ from 1990 to 2016 , and deaths have increased by $94 \%$, with contributing factors including population growth and aging [5].

Although MM accounts for only a relatively small percentage of all cancer types, the costs associated with its management are considerable. However, economic data on
MM are limited, and the available studies mainly focus on healthcare costs [9].

The main objective of this study was to determine the burden and cost of MM in Portugal from the perspective of the National Health Service (NHS), providing evidence to healthcare policy makers to support future decision making regarding disease management.

\section{Methods}

We determined the burden and cost of MM in Portugal using recent data provided by national data sources. These two components provided the disease impact, measuring the number of deaths, loss of health and economic impact of MM.

\subsection{Burden of Disease}

Burden of disease was assessed considering the impact in terms of DALYs, a metric also adopted by the World Health Organization (WHO) that measures the years of healthy life lost due to disease or premature death. It includes two timebased indicators: years of life lost (YLL), which measures premature mortality due to the disease; and years lost due to disability (YLD), which measures the number of years patients live with the disease $[11,12]$. Equation 1 is used to estimate DALYs,

DALYs $=$ YLL + YLD.

Equation 2 is used to estimate the YLLs of a population during a given period,

$\mathrm{YLL}=n \times L$,

where $n$ is the number of deaths in the population and $L$ is the average remaining life expectancy of the population, in years, at the age of death.

YLL was estimated based on MM Portuguese mortality data from 2018. Hospital-related mortality was assessed using data for Portugal from the European Cancer Information System (ECIS) [13], which was also the source of age of death data for 2018. These estimates were calculated based on the data submitted by the Portuguese National Cancer Registry, including data on life expectancy in Portugal (data on the resident population of Portugal, from the Portuguese Institute of Statistics).

YLD in the population was estimated based on a prevalence-based model, using Eq. 3 .

$\mathrm{YLD}=P \times W$, 
where $P$ is the number of prevalent cases and $W$ is the disability weight associated with the condition [14].

The prevalence of MM was estimated based on an analysis of the number of unique patients that generated production (e.g., hospitalizations, day care sessions) in all hospitals of the Portuguese NHS through patient diagnoses codified by the International Classification of Diseases, Ninth Edition. Thus, the calculated prevalence represents patients with MM diagnosed and treated in Portugal (patients with monoclonal gammopathy of undetermined significance or smoldering MM are typically asymptomatic and do not receive any treatment outside clinical trials).

We analyzed the NHS hospital diagnosis-related group (DRG) 2016 database. Regular data management procedures were developed to ensure data quality and anonymization. Patients with MM were identified using the relevant diagnosis codes, with patients recorded under any of the following codes considered in the analysis:

- C900.0: MM not having achieved remission.

- C900.1: MM in remission.

- C900.2: MM in relapse.

- C888: Other malignant immunoproliferative diseases.

- C889: Malignant immunoproliferative disease, unspecified.

The use of these codes identified 1941 patients, accounting for the prevalence of patients with MM treated in Portuguese hospitals. Table 1 summarizes the key patient characteristics.

The disability weight associated with a disease is quantified between 0 (no disability; perfect health) and 1 (total disability or death). Disability weights for MM were originally defined by expert panels of the WHO and re-estimated for the Global Burden of Disease Study 2016 through a largescale empirical investigation [5]. Disability weights were distributed according to four different groups of patients based on the type of treatment: diagnosis/first-line treatment; remission; relapse; terminal. Portuguese patients with MM were distributed among these treatment groups by an expert panel of Portuguese hematologists (see Table 2).

The length of disability for MM reflected an overall survival of 73.2 months, as reported by Kumar et al. [15], as we considered this value to be representative of the Portuguese population.

A local expert panel comprising six leading Portuguese hematologists from different hospitals and regions of the country gathered for an expert panel meeting on 6 June 2019 (Table 3). The meeting followed a modified Delphi method to obtain consensus on the results presented.

To achieve the objectives of this study and bearing in mind the lack of data available in Portugal on the incidence
Table 1 Characteristics of patients with multiple myeloma

\begin{tabular}{|c|c|c|}
\hline \multicolumn{2}{|l|}{ Characteristics } & \multirow{2}{*}{$\begin{array}{l}n(\%) \\
1941\end{array}$} \\
\hline Population & & \\
\hline \multirow[t]{2}{*}{ Sex } & Male & $1047(54)$ \\
\hline & Female & $894(46)$ \\
\hline \multirow[t]{8}{*}{ Age (years) } & $<55$ & $178(9)$ \\
\hline & $55-59$ & $140(7)$ \\
\hline & $60-64$ & $226(12)$ \\
\hline & $65-69$ & $279(14)$ \\
\hline & $70-74$ & $326(17)$ \\
\hline & $75-79$ & 331 (17) \\
\hline & $80-84$ & 269 (14) \\
\hline & $>85$ & $192(10)$ \\
\hline \multirow[t]{13}{*}{ District of residence } & Lisbon & $531(27)$ \\
\hline & Oporto & $243(13)$ \\
\hline & Aveiro & $152(8)$ \\
\hline & Setúbal & $146(8)$ \\
\hline & Coimbra & $116(6)$ \\
\hline & Leiria & $116(6)$ \\
\hline & Braga & $113(6)$ \\
\hline & Viseu & $95(5)$ \\
\hline & Santarém & $88(5)$ \\
\hline & Faro & $65(3)$ \\
\hline & Vila Real & $51(3)$ \\
\hline & Castelo Branco & $36(2)$ \\
\hline & Others & 189 (10) \\
\hline
\end{tabular}

Table 2 Patient distribution per disability weight — expert panel input

\begin{tabular}{llc}
\hline Stage & Disability weight & $\begin{array}{l}\text { Patient } \\
\text { distribution } \\
(\%)\end{array}$ \\
\hline Diagnosis/first-line treatment & 0.288 & 15 \\
Remission & 0.049 & 60 \\
Relapse & 0.451 & 24 \\
Terminal & 0.540 & 1 \\
Disability-weighted average & 0.186 & 100 \\
\hline
\end{tabular}

of MM, we analyzed the longitudinal retrospective hospital patient dataset of IQVIA Consulting. This dataset contained data for the 5-year period 2014-2018 for all drug-related consumption by patients with MM in 16 public hospitals, covering $43 \%$ of all patients treated within the Portuguese NHS. The incidence was determined by analyzing patient drug consumption in hospitals. Patients were classified as "naïve" if they had started treatment in 2018 and had not received any MM-related drugs throughout the retrospective 60-month data. Patients classified as naïve for 2018 were thus considered new cases of MM in 2018. These new patients were then extrapolated to the full population 
Table 3 Members of the expert panel

\begin{tabular}{lll}
\hline Expert & Hospital & Location \\
\hline Dr. Catarina Geraldes & Centro Hospitalar e Universitário de Coimbra & Coimbra \\
Dr. Cristina João & Clinical Center of the Champalimaud Center for the Unknown & Lisbon \\
Dr. Fernanda Trigo & Centro Hospitalar S. João & Oporto \\
Dr. Manuel Neves & Clinical Center of the Champalimaud Center for the Unknown & Lisbon \\
Dr. Paulo Lúcio & Clinical Center of the Champalimaud Center for the Unknown & Lisbon \\
Dr. Rui Bergantim & Centro Hospitalar S. João & Oporto
\end{tabular}

considering drug consumption in other NHS hospitals. The estimated incidence of MM in Portugal was 7.8/100,000 for 2018.

\subsection{Cost of Illness}

The cost of MM was estimated using a prevalence-based model, which estimated direct costs from the NHS perspective [16]. The cost for each item was calculated by multiplying the total amount of resources used by the unit costs of that resource. Unit costs were obtained from the official national tariffs [17], national cost lists of DRGs [20], and EHN (National Hospital Drugs Study) IQVIA databases. All the direct healthcare and non-healthcare costs associated with the disease that we were able to calculate were considered from the perspective of the NHS.

The model considered direct healthcare costs associated with MM, which can be divided into healthcare and non-healthcare costs. The former includes costs associated with diagnosis, hospitalizations (including hematopoietic stem-cell transplants), surgeries, emergency visits, medical appointments and drug usage and administration. Transportation costs were the only direct non-healthcare costs that we were able to determine. These costs are relevant since several patients live outside the district in which the hospital is located and so must travel to obtain the best treatment. Indirect costs were excluded from these analyses as they affect stakeholders other than the NHS and because data available to support such analysis in Portugal are scarce.

To quantify hospital production for patients with MM, we analyzed the NHS hospital DRG database from 2016 for all patients diagnosed, as previously defined.

The 1941 patients with MM that we identified generated 12,666 recorded hospital episodes in 2016, which were allocated across 178 different DRGs. The episodes were analyzed by medical experts, and those not directly or indirectly caused by MM were excluded from the analysis. Thus, a total of 11,878 episodes related to MM or disease comorbidity were considered for the cost quantification and allocated across 36 different DRGs, as shown in Table 4.
Table 4 Hospital production regarding patients with multiple myeloma

\begin{tabular}{lr}
\hline Hospital data & \multicolumn{1}{l}{$n$} \\
\hline Episodes & \\
Planned & 10,717 \\
Emergency & 1161 \\
Procedures & 21,480 \\
Inpatient days & 31,127 \\
\hline
\end{tabular}

\subsubsection{Direct Healthcare Costs}

This study considered direct costs recorded in hospital settings for the 1941 patients identified, representing all patients with MM diagnosed and treated in NHS hospitals in Portugal in 2016. We used this year for the data because it was the most recent complete year available for analysis. The costs associated with the episodes considered were valued according to the prices established by Portuguese Law (Portaria 254/2018) [18]. The national DRG funding value was used as a proxy for the hospital costs of treating these patients. These values vary by DRG code, the level of severity of the episode, and whether the treatment is outpatient or inpatient [20]. Direct healthcare included the following components.

2.2.1.1 Hospitalization All events codified with a medical DRG according to the NHS DRG database were considered. DRGs for chemotherapy sessions were excluded as they were costed separately under the MM-related drugs category. Hematopoietic stem-cell transplants were also included in this category even though they were coded as a surgical DRG.

2.2.1.2 Surgery Surgical DRGs codified for the selected patient pool were quantified, indicating that a given patient underwent a surgical procedure.

2.2.1.3 Healthcare Professional Visits The average number of hospital visits to healthcare professionals (HCPs) (hematologists only) by patients with MM was estimated for the different treatment groups of patients as determined by the local expert panel. Visits were estimated as follows: (1) patients not having achieved remission (initial diagnosis)_- 
estimated as having three visits during a 2-month period; (2) patients in remission-estimated as having 11 visits within a 1-year period; (3) patients in relapse-estimated as having eight visits during a 3-month period.

2.2.1.4 Emergency Visits The average number of emergency hospital visits by patients with MM was estimated according to the treatment group of patients as determined by the local expert panel: (1) patients not in remission (initial diagnosis)—estimated as having one visit during a 2-month period; (2) patients in remission-estimated as having one visit within a year; and (3) patients in relapseestimated as having 2.5 visits during a 3 -month period.

Emergency visits leading to hospitalization were not considered in this category as they were already included under "hospitalizations". Only emergency episodes that did not result in an inpatient episode were costed.

\subsubsection{Multiple Myeloma (MM)-Related Drugs Drug} costs were analyzed using the MM-related molecule consumption in 2018. The data source used was the EHN (National Hospital Drugs Study) database of the IQVIA, which includes annual national hospital molecule consumption. The molecules for which costs were considered included bortezomib, carfilzomib, ixazomib, daratumumab, elotuzumab, thalidomide, lenalidomide and pomalidomide. Molecules without an exclusive indication for MM were considered with an "MM-attributable" ratio. MM treatment was the only indication for all the selected molecules, apart from lenalidomide, for which it was considered that $99 \%$ of total molecule consumption was associated with $\mathrm{MM}$; the other $1 \%$ of indications was associated with myelodysplastic syndrome with del $(5 q)$ or chronic lymphatic leukemia and other B-cell non-Hodgkin lymphomas.

The NHS payment price for the administration of intravenous/subcutaneous drugs was added as a proxy for administration cost [20].

2.2.1.6 Exams (Diagnostic and Follow-Up) The costs for diagnostic exams, such as laboratory testing and imaging, were already included in the funding prices paid by the NHS for HCP visits, inpatient and outpatient surgery episodes, medical hospitalization episodes and emergency visits. Therefore, in this study, the cost for diagnostic procedures was included but not costed separately.

\subsubsection{Direct Non-Healthcare Costs}

This study estimated costs associated with transportation of patients with MM for HCP visits for consultations, emergency visits, treatment sessions, surgery and hospitalizations. The NHS hospital DRG database for hospital production contains data on patients' residential districts and the hospitals visited, enabling quantification of the number of patients treated outside their district of residence. The transportation costs were then calculated based upon two possible scenarios: (1) patient's residence and treatment hospital in the same district and (2) patient's residence and treatment hospital not in the same district.

The maximum NHS payment price for these two scenarios varied according to the mode of transport used by the patient, i.e., ambulance versus private vehicle [19]. The distribution between the type of vehicle used was defined by the expert panel, as shown in Table 5.

Where the patient's residence and the treatment hospital were not in the same district, the district hospital closest to the patient's home address was used. The average cost per visit was then calculated according to the number of selected episodes recorded. This average cost was applied for the transportation of all hospital visit types, apart from emergency visits, where the price considered was always that of an ambulance.

\section{Results}

\subsection{Burden of Disease}

MM is a disease with high mortality and morbidity. We calculated that, in 2018, there were 8931 DALYs in Portugal due to MM. This result reflects a total of 8570 YLLs and 361 YLDs in the Portuguese population. These results are summarized in Table 6.

Most of the impact resulted from YLLs due to premature death, which resulted in 8570 DALYs, representing 96.0\% of the total DALYs. According to the ECIS, hospital-related mortality for Portugal in 2018 was 6.9/100,000, with a median age of death of 75 years [13, 20], whereas the life expectancy in Portugal for the same period was 77.7 years for men and 83.4 years for women.

MM also caused a loss of 361 DALYs due to disability, representing $4.0 \%$ of the total DALYs. The YLDs were obtained considering a prevalence of 1941, as shown in the NHS database. Based on these data, the estimated MM prevalence for Portugal's resident population was 18.9/100,000. Its incidence was estimated at 7.8/100,000.

Table 5 Hospital visits for patients with multiple myeloma per transport type-expert panel input

\begin{tabular}{llll}
\hline Transport situation & Units & Ambulance & Private vehicle \\
\hline Not in same district & Price per km & $€ 0.51$ & $€ 0.35$ \\
Same district & Fixed price & $€ 7.5$ & $€ 10$ \\
Expert panel distribution $(\%)$ & 70 & 30 \\
\hline
\end{tabular}


Table 6 Disability-adjusted life-years for multiple myeloma in Portugal

\begin{tabular}{lll} 
DALYs $(8570+361=8931)$ & $\begin{array}{l}\text { YLLs (mortality per age interval } \times \text { average remaining } \\
\text { life expectancy of the population, in years, at the age } \\
\text { of death) }\end{array}$ & $\begin{array}{c}\text { YLDs }(\text { prevalence } \times \text { disability weight }=1941 \times 0.186= \\
361 \text { DALYs })\end{array}$ \\
$8931(100 \%)$ & $8570(96.0 \%)$ & $361(4.0 \%)$ \\
\hline
\end{tabular}

$D A L Y s$ disability-adjusted life-years, $Y L D s$ years lost due to disability, $Y L L s$ years of life lost

According to the expert panel, which estimated the distribution of patients with MM according to treatment groups, the average disability weight considered was 0.186 . The length of disability for MM was considered as an overall survival of 73.2 months, as reported by Kumar et al. [10].

\subsection{Cost of Illness}

Total costs associated with patients with MM in Portugal amounted to $€ 61,041,673$ in 2018, resulting in an average cost of $€ 31,449$ per patient/year, with pharmacological treatment accounting for $81 \%$ of the total. A breakdown of the results for each component, in relation to direct healthcare and non-healthcare, is given in Table 7.

\subsubsection{Direct Healthcare Costs}

Considering the analysis of the 1941 patients with MM, we identified the following direct costs in 2018.
3.2.1.1 Medical Hospitalizations Of the 12,355 medical DRGs identified, 1631 were included in the cost quantification (10,098 episodes encoded as "chemotherapy session" were excluded as pharmacological costs and considered separately). The total cost for the medical episodes considered was $€ 8,225,626$, an average of $€ 4238$ per patient/year. This includes 188 hematopoietic ASCT episodes, accounting for a total of $€ 3,325,975$.

3.2.1.2 Surgeries Of the 311 surgical DRGs identified, 149 were included in the cost quantification and considered MM related. The total cost for the surgical episodes considered was $€ 643,827$, an average of $€ 332$ per patient/year.

3.2.1.3 Hospital Visits to Healthcare Professionals The HCP list price is indexed by hospital group. The authors considered a weighted HCP visit price, which takes into account the distribution of the 11,878 included episodes by hospital categories [17]. This resulted in a total of 24,238 HCP appointments in 1 year, with a total cost of $€ 1,706,631$ and an average cost of $€ 879$ per patient/year.
Table 7 Multiple myeloma direct healthcare and nonhealthcare costs in 2018

\begin{tabular}{lllcr}
\hline Variables & $\begin{array}{l}\text { Average annual } \\
\text { quantity of } \\
\text { resources }(n)\end{array}$ & $\begin{array}{l}\text { Weighted } \\
\text { average unit } \\
\text { cost }(€)\end{array}$ & $\begin{array}{l}\text { Average cost per } \\
\text { patient/year }(€) ; 1941 \\
\text { patients }\end{array}$ & $\begin{array}{r}\text { Total costs }(€) ; \\
1941 \text { patients }\end{array}$ \\
\hline Hospital visits & 24,238 & 70 & 879 & $1,706,631$ \\
Emergency visits & 2114 & 50 & 54 & 105,722 \\
Surgery & 149 & 4320 & 332 & 643,827 \\
Medical hospitalization & 1631 & 5043 & 4238 & $8,225,626$ \\
Pharmacologic treatment & & & & \\
Lenalidomide & NA & NA & 14,110 & $27,386,548$ \\
Pomalidomide & NA & NA & 1163 & $2,257,453$ \\
Thalidomide & NA & NA & 1010 & $1,959,456$ \\
Bortezomib & NA & NA & 5348 & $10,380,969$ \\
Carfilzomib & NA & NA & 777 & $1,508,388$ \\
Daratumumab & NA & NA & 2835 & $5,502,830$ \\
Administration & 10,098 & 20 & 105 & 203,980 \\
Total direct healthcare & & & 30,851 & $59,881,429$ \\
Transportation & NA & 30 & 598 & $1,160,244$ \\
Direct non-healthcare & & & 598 & $1,160,244$ \\
Total direct costs & & & 31,449 & $61,041,673$ \\
\hline
\end{tabular}

NA not applicable 
3.2.1.4 Emergency Visits The average number of hospital visits to the emergency room (ER) per patient was determined by the expert panel. This resulted in a total of 3275 ER visits in 1 year. However, 1161 episodes with urgent admission were then excluded because this cost was already included in the hospitalization DRGs. The remaining $2114 \mathrm{ER}$ visits were then assumed not to have resulted in an inpatient episode. This led to a total ER visit cost of $€ 105,722$ and an average cost of $€ 54$ per patient/ year.

3.2.1.5 Drugs Therapeutics for MM have evolved over the years. The total cost for pharmacological treatment considered was $€ 49,199,624$, including $€ 203,980$ for drug administration, an average of $€ 25,348$ per patient/year. MM-related hospital consumptions in 2018 were as follows:

- Oral therapy considered lenalidomide ( $€ 27,386,548)$, pomalidomide $(€ 2,257,453)$ and thalidomide $(€ 1,959,456)$, representing $64.2 \%$ of total drug costs.

- Parenteral agents Bortezomib $(€ 10,380,969)$, carfilzomib $(€ 1,508,388)$ and daratumumab $(€ 5,502,830)$, representing $35.4 \%$ of total drug costs. Since the available forms of these drugs are indicated for intravenous or subcutaneous use, the administration costs were also considered. The NHS payment price for the administration of perfusion drugs was $€ 20.20$ for each service. This fee was applied to the 10,098 episodes encoded as "chemotherapy session" in 2016, generating a total administration cost of $€ 203,980$ and representing $0.4 \%$ of total costs.

3.2.1.6 Exams (Diagnostic and Follow-up) As discussed, the cost for diagnostic procedures was included but not costed as an isolated item.

\subsubsection{Direct Non-Healthcare Costs}

3.2.2.1 Transportation Transportation to and from medical and surgical episodes, chemotherapy sessions and HCP appointments were calculated according to the weighted unique transportation value (apart from emergency visits, where the price considered was the cost of an ambulance).

This resulted in a total transportation cost of $€ 1,160,244$, with an average of $€ 598$ per patient/year.

It should be noted that the three major hospitals are located in Lisbon, Oporto and Coimbra, with 30\%, 37\% and $66 \%$ of their patients, respectively, traveling from other districts, which explains the high associated transportation costs.

\section{Discussion}

The main purpose of this study was to provide an estimate of the burden of disease in terms of DALYs and costs to the NHS. The results provide a clear demonstration of the impact of MM in Portugal.

When addressing a disease with an increasing incidence rate in an aging population, it is essential to discuss the context of MM in Portugal. MM does not have as many DALYs (8931) as diabetes $(181,197)$ or atrial fibrillation $(18,879)$, which are high-prevalence diseases with considerable impact in society. However, the MM ratio of DALY to prevalence yields is $4: 9$, which is higher than those of diabetes and atrial fibrillation (0:8 and 2:6, respectively). This comparison demonstrates the considerably higher burden of disease per patient with MM than with diabetes or atrial fibrillation [21-23].

When analyzing the results of the burden of disease for $\mathrm{MM}$, it can be concluded that most DALYs are due to YLLs rather than YLDs, a conclusion that was expected and is consistent with the results from the Global Burden of Disease Study, which estimated 9157 DALYs in 2016 with a similar distribution. The YLL value for MM is due to the high mortality rate of the disease in Portugal of 6.9/100,000, one of the highest in Europe [5, 13], with significantly reduced life expectancy among patients up to the age of 75 years.

Our research enabled us to estimate the direct cost of the disease as $€ 61,041,673$ in 2018, with MM pharmacological treatment accounting for $81 \%$ of the total. This result is relevant because no formal estimate of the total direct costs attributable to MM in Portugal has been available until now. These costs are higher than the cost per patient of $€ 16,149$ reported by Petrucci et al. [24] for Italy (vs. the current cost in Portugal of $€ 31,449$ ). This difference is mainly because the Petrucci et al. [24] study was conducted in 2008, when most products for the treatment of MM were not widely available. However, these costs are expected to increase with the earlier use of novel drugs in MM treatment, with better efficacy and more prolonged survival outcomes. When compared with other burdensome diseases in Portugal, the per-patient direct cost is much higher than the $\sim € 1300$ per patient with diabetes or the $\sim € 830$ per patient with atrial fibrillation, which highlights the relevance of this disease [21-23].

Analyzing direct costs excluding pharmacological treatments, we estimated a total of $€ 5503 /$ patient. Antunes et al. [9] reported a higher value of $€ 10,139 /$ patient for 2015. This difference may be because this study was performed at a central hospital, where ASCT costs accounted for $32 \%$ of the total. By contrast, in the present study, ASCT represents only $9 \%$ of the total amount. Nevertheless, the total direct healthcare costs reported in this 
study are comparable to those reported by Antunes et al. [9], at $€ 26,647$ (or $€ 27,562$, considering the 2018 annual inflation rate).

Analysis of pharmacological treatment through national molecule consumption enabled us to calculate the approximate cost of a chemotherapy session. When considering only parenteral drugs (bortezomib, carfilzomib and daratumumab) plus the cost of their administration, the total cost was $€ 17,596,167$. Given the 10,098 chemotherapy DRG-coded episodes, the average cost of a chemotherapy session is $€ 1743$. But the NHS DRG funding table provides only $€ 496$ per outpatient episode. This analysis underlines the fact that the DRG table may underestimate the costs used as a proxy for the actual price of medical procedures.

For methodological reasons, the results presented in this study may underestimate the cost and burden of the disease, since they do not include any indirect costs, such as those derived from patients' and informal caregivers' absences from work or early retirement. Another factor is the number of patients with MM, as the incidence was calculated to include non-treated patients who started medication in 2018 and had not received any MM-related drugs throughout the retrospective 60 -month data. Thus, the number of new patients might be underestimated if some patients were in remission for a period longer than the study duration.

Moreover, this study included only diagnosed and treated patients, as patients recorded in IQVIA databases were considered as the main source. Costs resulting from underdiagnosis, costs incurred before diagnosis and any costs for patients with indolent MM were not considered. The IQVIA data only include NHS hospitals, so privately treated patients were not included in this analysis. Hence, our results only reflect the NHS perspective, and payments by patients themselves or other third parties were not considered.

We used the most recent NHS hospital production database available for the cost calculations, referring to episodes occurring in 2016. The limitation of the timeliness of the DRG data compared with every other epidemiological indicator was mitigated by the principle that clinical practice (in terms of hospital production-exams, diagnosis, etc.—excluding pharmacological treatment) remained largely consistent and that using 2018 DRG table prices would give the approximate cost of 2018 hospital production. The most significant difference in cost would lie in pharmacological treatment, with more innovative treatments being used in the past 2 years, so this limitation was avoided by using data from 2018.

\section{Conclusion}

With the increasing prevalence of MM in recent years, and the increased costs alongside treatment innovation, it is necessary to generate data on this disease at a national level.

MM is a disease with a high burden. Most DALYs are due to YLLs rather than YLDs, because of the high mortality rate in Portugal, one of the highest in Europe. Most of the estimated cost of the disease ( $€ 61$ million in 2018) is associated with pharmaceuticals, of which $77 \%$ is attributable to lenalidomide and bortezomib. These costs are expected to rise as more effective drugs become available and overall patient survival increases.

Despite some limitations, this study provides updated insights into the costs associated with MM for most of the Portuguese population. These results will hopefully raise awareness of the disease and prompt new scientific research into, and more investment in the healthcare management of, this high-impact disease.

\section{Declarations}

Author Contributions RB, MN, MN HP and PL collected data, designed and developed the analysis and wrote and reviewed the manuscript. CG, CJ, FT and JM critically reviewed the analysis of the data and its design and reviewed the manuscript.

Funding This study was developed by the Portuguese Group of Multiple Myeloma (Grupo Português de Mieloma Múltiplo) and funded by Janssen Portugal.

Conflict of Interest Manuel Neves has received honoraria for speaking and/or participation in advisory boards for Janssen, Takeda, Amgen and Celgene. Fernanda Trigo has received honoraria for speaker services from Takeda, Amgen, Celgene and Janssen and for attendance at advisory boards from Takeda, Amgen, Celgene and Janssen. Rui Bergantim has received research grants from APCL/SPH/AMGEN and Celgene and honoraria for speaking and/or participation in advisory boards from Celgene, Janssen, Takeda and Amgen. Cristina João has received a research grant from Takeda and honoraria for speaking and/ or participation in advisory boards from Celgene, Janssen, Takeda, AbbVie and Amgen. Paulo Lúcio has received honoraria for speaking and/or participation in advisory boards from Celgene, Janssen, Takeda and Amgen. Mariana Neves and Hugo Pedrosa are employees of IQVIA Solutions Portugal. João Mendes is an employee of Janssen Cilag Farmacêutica, Portugal. Catarina Geraldes has received honoraria from Janssen, Celgene, Amgen, Gilead and Takeda for lectures and participation in advisory boards.

Data Availability Data used for this study relied on two main data sources to support the quantification of the burden and cost of MM: the NHS 2016 DRG dataset and the 2013-2018 drug longitudinal retrospective hospital patient dataset of IQVIA Consulting. These datasets are not publicly available and were acquired for the development of this study. Data used are presented in aggregate in this paper. Other public data sources used in the development of this study are disclosed in the reference list. 
Open Access This article is licensed under a Creative Commons Attribution-NonCommercial 4.0 International License, which permits any non-commercial use, sharing, adaptation, distribution and reproduction in any medium or format, as long as you give appropriate credit to the original author(s) and the source, provide a link to the Creative Commons licence, and indicate if changes were made. The images or other third party material in this article are included in the article's Creative Commons licence, unless indicated otherwise in a credit line to the material. If material is not included in the article's Creative Commons licence and your intended use is not permitted by statutory regulation or exceeds the permitted use, you will need to obtain permission directly from the copyright holder. To view a copy of this licence, visit http://creativecommons.org/licenses/by-nc/4.0/.

\section{References}

1. Institute, I. (2017) Medicines Use and Spending in the US.

2. Venkatesan P. Cancer-related productivity losses in BRICS countries. Lancet Oncol. 2018;19:e147. https://doi.org/10.1016/S1470 -2045(18)30090-1.

3. Lopes JM, Gonçalves FR, Borges M, Redondo P, Laranja-Pontes J. The cost of cancer treatment in Portugal. Ecancermedicalscience. 2017;11:1-10. https://doi.org/10.3332/ecancer.2017.765.

4. Moreau P, San Miguel J, Sonneveld P, Mateos MV, Zamagni E, Avet-Loiseau H, et al. Multiple myeloma: ESMO Clinical Practice Guidelines for diagnosis, treatment and follow-up. Ann Oncol. 2017;28:iv52-61. https://doi.org/10.1093/annonc/mdx096.

5. Cowan AJ, Allen C, Barac A, Basaleem H, Bensenor I, Curado MP, et al. Global burden of multiple myeloma. JAMA Oncol. 2018;4:1221. https://doi.org/10.1001/jamaoncol.2018.2128.

6. Kumar SK, Callander NS, Alsina M, et al. NCCN Guidelines Insights: multiple myeloma, version 3. 2018. J Natl Compr Cancer Netw. 2018;16:11-20. https://doi.org/10.6004/jnccn.2018.0002.

7. Ashcroft J, Duran I, Hoefeler H, et al. Healthcare resource utilisation associated with skeletal-related events in European patients with multiple myeloma: Results from a prospective, multinational, observational study. Eur J Haematol. 2018;100:479-87. https:// doi.org/10.1111/ejh.13044.

8. Terpos E, Kleber M, Engelhardt M, et al. European myeloma network guidelines for the management of multiple myeloma-related complications. Haematologica. 2015;100:1254-66. https://doi. org/10.3324/haematol.2014.117176.

9. Antunes L, Rocha-Gonçalves F, Chacim S, Lefèvre C, Pereira M, Pereira S, et al. Real-world treatment patterns, resource use and cost burden of multiple myeloma in Portugal. Eur J Cancer Care. 2019. https://doi.org/10.1111/ecc.13026.

10. Lea RA. World development report 1993: 'Investing in Health.' Milton: Taylor \& Francis; 1993.

11. Murray CJL. Global burden of disease Le poids de la morbidite dans le monde quantifying the burden of disease : the technical basis for disability-adjusted life years. Bull World Health Organ. 1994;72:429-45. https://doi.org/10.1016/S0140-6736(96)07495 $-8$.

12. Schroeder SA. Incidence, prevalence, and hybrid approaches to calculating disability-adjusted life years. Popul Health Metr. 2012;10:19. https://doi.org/10.1186/1478-7954-10-19.

13. ECIS-European Cancer Information System. Estimates of cancer incidence and mortality in 2018, for all countries. 2018. https:// ecis.jrc.ec.europa.eu/index.php.

14. Chen A, Jacobsen KH, Deshmukh A, Cantor SB. The evolution of the disability-adjusted life year (DALY). Soc Econ Plan Sci. 2015;49:10-5. https://doi.org/10.1016/j.seps.2014.12.002.

15. Kumar SK, Dispenzieri A, Lacy MQ, et al. Continued improvement in survival in multiple myeloma: changes in early mortality and outcomes in older patients. Leukemia. 2014;28:1122-8. https ://doi.org/10.1038/leu.2013.313.

16. Clabaugh G, Ward MM. Cost-of-illness studies in the United States: a systematic review of methodologies used for direct cost. Value Health. 2008;11:13-21.

17. Saúde Ministério. Circular Normativa N. 8/2018/DPS/ACSS. Hospital Framework Contract 2018:1-6. 2019. www.acss.min-saude .pt/wp-content/uploads/2018/07/Circular_Normativa_8_2018.pdf.

18. Diário da República. Portaria no. 254/2018. NHS Price lists. 2018. https://dre.pt/home/-/dre/116353279/details/maximized.

19. Saúde Ministério. Despacho no. 7702-A/2012. 2012: 20410. NHS Transport fees. 2012.

20. World Health Organization (WHO). International Agency for Research on Cancer. Estimated number of deaths in 2018, multiple myeloma, both sexes, all ages. Cancer Today. 2018:2018. https ://gco.iarc.fr/today/home.

21. Diabetes ON. Relatório do Observatório Nacional da Diabetes. 2016.

22. Evaluation, I. for H.M. and. Global Burden of Disease Compare. 2020.

23. Gouveia M, Costa J, Alarcão J, et al. (2015) Burden of disease and cost of illness of atrial fibrillation in Portugal. Rev Port Cardiol. 2015;34(1):1-11.

24. Petrucci MT, Calabrese E, Levi A, et al. Cost of illness in patients with multiple myeloma in Italy: the CoMiM study. Tumori. 2013;99:e193-202. https://doi.org/10.1177/030089161309900434. 